\title{
The effect of positive changes during intraoperative monitoring of the functional improvement in patients with cervical compressive myelopathy
}

This article was published in the following Dove Press journal:

Clinical Interventions in Aging

\author{
Min Kyu Park' \\ Sook Joung Lee ${ }^{2}$ \\ Sang Beom Kim ${ }^{3}$ \\ Kyeong Woo Lee $^{3}$ \\ Hye-Jeong Lee ${ }^{4}$ \\ Eun Young $\mathrm{Han}^{5}$ \\ Bo Ryun Kim ${ }^{5}$
}

'Department of Pharmacology and Clinical Pharmacology, Dong-A University College of Medicine and Hospital, Busan, Republic of Korea; ${ }^{2}$ Department of Physical Medicine and Rehabilitation, Catholic University of Korea, Daejeon St Mary's Hospital, Daejeon, Republic of Korea; ${ }^{3}$ Department of Physical Medicine and Rehabilitation, Dong-A University College of Medicine, Dong-A University Hospital, Busan, Republic of Korea; ${ }^{4}$ Department of Pharmacology, College of Medicine, Dong-A University, Busan, Republic of Korea; ${ }^{5}$ Department of Rehabilitation Medicine, Jeju National University School of Medicine, Jeju National University Hospital, Jeju, Republic of Korea
Correspondence: Sook Joung Lee Department of Physical Medicine and Rehabilitation, Catholic University of Korea, Daejeon St Mary's Hospital, Daeheung Ro 64, Jung-gu, Daejeon 34943, Republic of Korea

Tel +82 422209650

Fax +82 422209035

Email Isj9954I4@hanmail.net
Background: Cervical compressive myelopathy (CCM) is a progressive, degenerative spine disease and the most common cause of spinal cord dysfunction in older individuals. Current clinical guidelines for spinal surgery recommend multimodal intraoperative monitoring (IOM) during spinal surgery as a reliable and valid diagnostic adjunct to assess spinal cord integrity. The aim of this study was to evaluate the effect of positive changes during IOM on the functional status in patients with CCM.

Methods: Patients who underwent spinal surgery with IOM due to CCM were enrolled. During the surgery, patients underwent IOM using motor evoked potential (MEP) and somatosensory evoked potential (SEP). MEP and SEP were checked before and immediately after decompression. A decrease in latency $>10 \%$ or an increase in amplitude $>50 \%$ was regarded as a "positive changes". Subjects were divided according to the presence of positive changes. Motor scores of American Spinal Injury Association (ASIA) impairment scale and Korean version of Modified Barthel Index (K-MBI) were evaluated before and after operation.

Results: Twenty-nine patients underwent spinal surgery due to CCM. Eleven patients showed positive changes in MEP during IOM. When the two groups were compared, improvement rate in the ASIA motor score and K-MBI were significantly higher in patients with positive changes than in patients without positive changes at 1 month after surgery. However, 6 months after surgery, there were no significance differences between the groups. Regardless of positive change, nearly all patients suffered from neuropathic pain after operation.

Conclusion: Positive changes in MEP during IOM may affect functional improvement 1 month after operation and early discharge without significant complications in CCM patients. However, they do not affect the neuropathic pain and long-term functional outcome. Thus, tailored proper management is needed to achieve maximal functional recovery in each patient after cervical spinal decompression surgery.

Keywords: cervical myelopathy, degenerative disorder, intraoperative monitoring, function, positive change

\section{Introduction}

Cervical compressive myelopathy (CCM) is a progressive, degenerative spine disease and the most common cause of spinal cord dysfunction in older individuals worldwide. ${ }^{1}$ CCM is characterized by spinal cord compression due to physiologic narrowing of the sagittal diameter of the spinal canal secondary to degenerative changes in the cervical spine. ${ }^{2}$ Its clinical presentation includes numbness, weakness, neck pain, loss of dexterity, and problems with gait. ${ }^{1,2}$ Recently, intraoperative monitoring (IOM) has been increasingly used to prevent neurological impairment during spinal cord surgery. ${ }^{3,4}$ 
Changes during IOM are also associated with corresponding spinal cord functions during surgery. ${ }^{5}$

Current clinical guidelines for spinal surgery recommend multimodal IOM including somatosensory evoked potential (SEP) and motor evoked potential (MEP) during spinal surgery as a reliable and valid diagnostic adjunct to assess spinal cord integrity. ${ }^{5}$ Originally, the purpose of IOM was to prevent neurological complications during surgical procedure, such as the resection of brain and spinal lesions. Thus, negative changes such as increase in latencies $>10 \%$ and decrease in amplitudes $>50 \%$ have been defined as "warning signs" indicative of postoperative neurological complication. ${ }^{6,7}$

Rather than focusing on warning signs, recently, Wang et al revealed that CCM patients have favorable prognosis in terms of neurological recovery when they demonstrate improvements in intraoperative MEP during cervical spinal cord decompression. ${ }^{8}$ Several studies revealed that intraoperative MEP can be used to detect the functional integrity of corticospinal tracts with high sensitivity and specificity. ${ }^{9}$

However, patients who have undergone cervical operation show various clinical manifestations and clinical outcomes according to their postoperative care. To the best of our knowledge, a few studies have evaluated the relation between changes during IOM and functional changes in CCM patients. Moreover, there is insufficient evidence regarding the therapeutic relationship between electrophysiological monitoring during spinal surgery and neurological outcomes. ${ }^{5}$ In addition, there are few previous studies regarding neuropathic pain and IOM changes. Thus, the primary aim of our study was to evaluate the effect of positive changes during IOM on the functional status in patients with CCM. The secondary aim was to explore whether it would also affect neuropathic pain.

\section{Methods}

\section{Subjects}

Patients with CCM who underwent spinal surgery with IOM at the Spine Center of Dong-A University Hospital were enrolled. Patients who had spinal stenosis without cord compression, spinal cord tumor, or metastatic spine lesion including leptomeningeal seeding, combined thoracic lesion, and previous CNS disease such as stroke or neurodegenerative disease were excluded. Patients with unreliable or missing preoperative MEP and SEP data were also excluded from this study. All patients demonstrated at least one clinical sign of myelopathy. One day before the spinal surgery, all patients underwent preoperative functional and neurophysiologic evaluation at the Department of Rehabilitation. Approximately 4 days after operation, all patients were consulted for physical therapy to the Department of Rehabilitation. Among these patients, those who needed intensive rehabilitation therapy were transferred to the Department of Rehabilitation.

\section{IOM}

Values of evoked potential were recorded using the ISIS IOM System $^{\circledR}$ (Inomed, Emmendingen, Germany). During the surgery, patients underwent IOM using MEP of abductor pollicis brevis and abductor halluces and SEP of median and tibial nerve. MEP and SEP were checked before and immediately after decompression. These variables were checked repeatedly if there were warning signs or if needed by the surgeon during decompression. During IOM, the latency and amplitude of evoked potentials on the upper and lower extremity were checked. According to a study by Wang et al, an increase in amplitude of $>50 \%$ and a decrease in latency of $>10 \%$ were defined as a "positive change". ${ }^{8}$ Subjects were divided according to the presence of positive changes into the "positive change" group and the "no positive change" group. All surgeries were conducted by experienced spine neurosurgeons and their surgical team, and IOM was completed and interpreted by veteran physiatrists.

\section{Anesthesia}

General anesthesia was performed using total intravenous anesthesia, such as Fresofol ${ }^{\circledR}$ (propofol; Fresenius Kabi, Seoul, Korea) or Remifentanil ${ }^{\circledR}$ (fentanyl; Hana Pharm, Seoul, Korea) based on hemodynamic response. No muscle relaxant or inhalation agent such as halothane was used during IOM. During anesthesia, all patients were maintained under normothermic and normotensive conditions.

\section{Functional evaluation}

Motor impairment was evaluated using the American Spinal Injury Association (ASIA) impairment scale, and functional evaluation was measured using the Korean version of Modified Barthel Index (K-MBI).

The ASIA score, established in 1973, is a neurological exam that is widely used to document sensory and motor impairments following spinal cord injury, and it is the gold standard for assessing spinal cord injury. ${ }^{10,11}$ The exam is based on the evaluation of the strength of muscles that control key motions on both sides of the body. Muscle strength is scored on a scale of $0-5$ according to a manual muscle test. ${ }^{12}$ The K-MBI was translated by six senior Korean physiatrists 
using the 5th version of the Modified Barthel Index; its reliability and validity was approved to assess activities of daily living. ${ }^{13}$

All functional evaluations were performed 1 day before the operation and at 1 month and 6 months after the operation by two physiatrists who were not aware of group allocation. The improvement rate in ASIA motor score and K-MBI was calculated using the following equation: (postoperative values - preoperative values)/preoperative values $\times 100 \%$. The improvement rate was measured by comparing values obtained before and at 1 month or 6 months after operation. The study protocol was approved by the Institutional Review Board of Dong-A University hospital, and all patients provided written informed consent.

\section{Statistical analysis}

Continuous data were presented as mean (SD) and binary data as number (percentage). Mann-Whitney $U$-test was used to compare baseline characteristics and the effectiveness of treatments between the two groups. The chi-squared test was used to compare sex, trauma, ASIA level, and neuropathic pain between the groups. Wilcoxon signed rank test and Friedman test were used to determine changes between values before and after treatment in each group. A $p$-value of $<0.05$ was considered statistically significant. SPSS 21.0 (SPSS, Inc., Chicago, IL, USA) for Windows was used for statistical analyses.

\section{Results}

A total of 29 patients were enrolled in this study. Among these patients, 11 showed positive changes in MEP amplitude and latency during IOM and were assigned to the "positive change" group. Two patients displayed "warning signs" in MEP amplitude during IOM but recovered within 1 hour. Figure 1 shows a case with a positive change (Figure 1A) and a case with a warning sign (Figure 1B) during IOM. None of the patients showed positive changes or warning signs in SEP during IOM. Thus, "positive change" group was determined according to changes in MEP only.

Table 1 shows the demographic characteristics of patients. Patients in the "positive change" group had a significantly shorter hospital stay than did patients in the "no positive change" group. Preoperative ASIA motor scores, K-MBI, and total operation duration were not significantly different between the groups. Regardless of positive change, nearly all patients $(90.9 \%$ in the positive change group and $100 \%$ in the no positive change group) suffered from neuropathic pain at 6 months after operation.
Table 2 presents the changes during IOM pre-decompression and post-decompression in each group. The positive change group showed significant improvements in MEP latency and amplitude but did not show significant changes in SEP variables.

In the positive change group, the ASIA motor scores showed significant improvements with time after operation relatively to that before operation. However, the "no positive change" group displayed a slight decrease in motor score at 1 month after operation relative to that before operation, but the motor score recovered at 6 months after operation (Figure 2A). Additionally, the K-MBI slightly decreased at 1 month after operation relative to that before operation, but it recovered and improved more at 6 months after operation in both groups (Figure 2B). Both groups displayed significant improvements in ASIA motor scores and K-MBI at 6 months after operation relative to that before operation.

When comparing improvement rates between the two groups, both ASIA motor score and K-MBI were significantly higher in patients in the positive change group than in patients in the no positive change group (Figure 3 ). However, 6 months after surgery, there were no significance differences in improvement rate between the two groups (Figure 3).

\section{Discussion}

This study focused on evaluating the relationship between positive changes during IOM and functional improvement in patients with CCM. Our results showed that positive changes in MEP during IOM affect functional improvement 1 month after operation and early discharge but does not affect functional improvement after 6 months. Changes in MEP were not associated with neuropathic pain.

\section{Relation between IOM during spinal surgery and functional outcomes}

There is insufficient evidence on the therapeutic relationship between IOM during spinal surgery and neurological outcomes. ${ }^{5}$ Previous studies on detethering cord surgery, cauda equina, or lumbar spinal stenosis showed that patients with an increase in MEP amplitude after decompression displayed a greater degree of postoperative improvement than patients without MEP increase. ${ }^{14-17}$ A few studies have evaluated positive changes during IOM and functional status in patients with CCM. Wang et al revealed that improvements in MEP amplitude after cervical spinal cord decompression indicate favorable prognosis in CCM patients. The authors emphasized that MEP monitoring can not only prevent neural injury but can also assess neural recovery. 
A

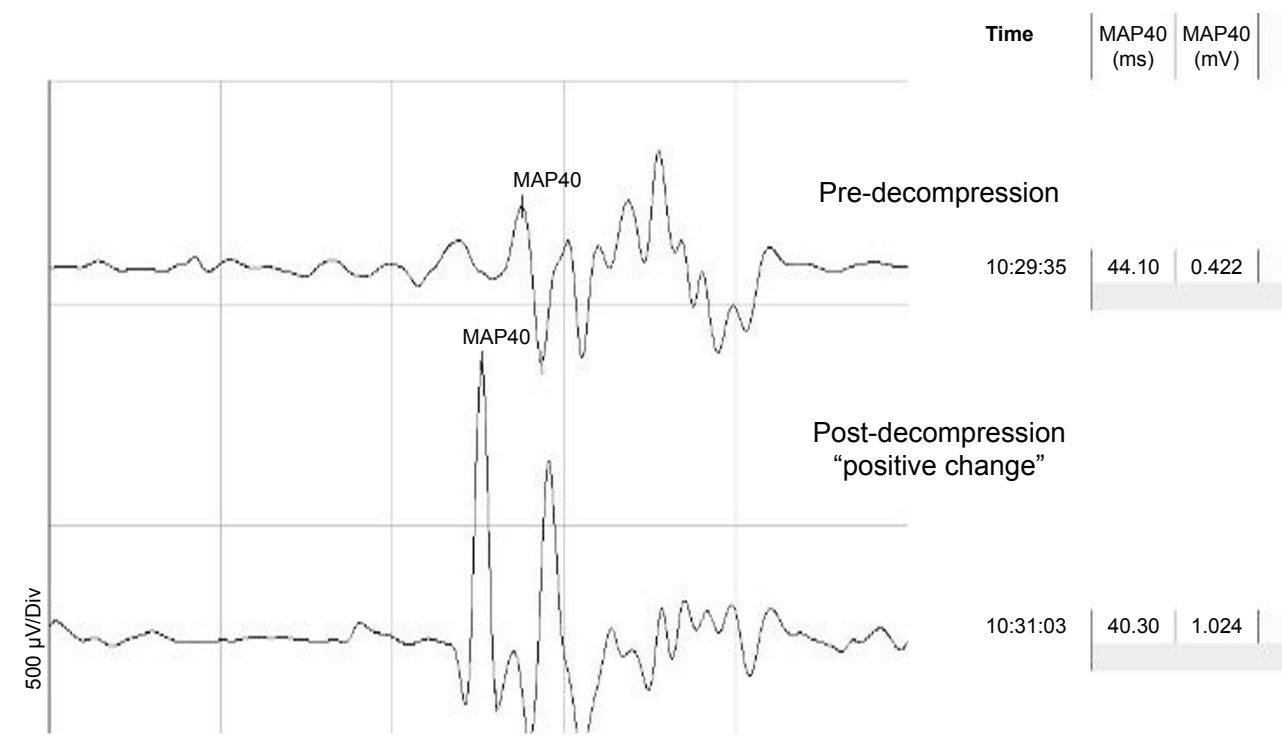

B

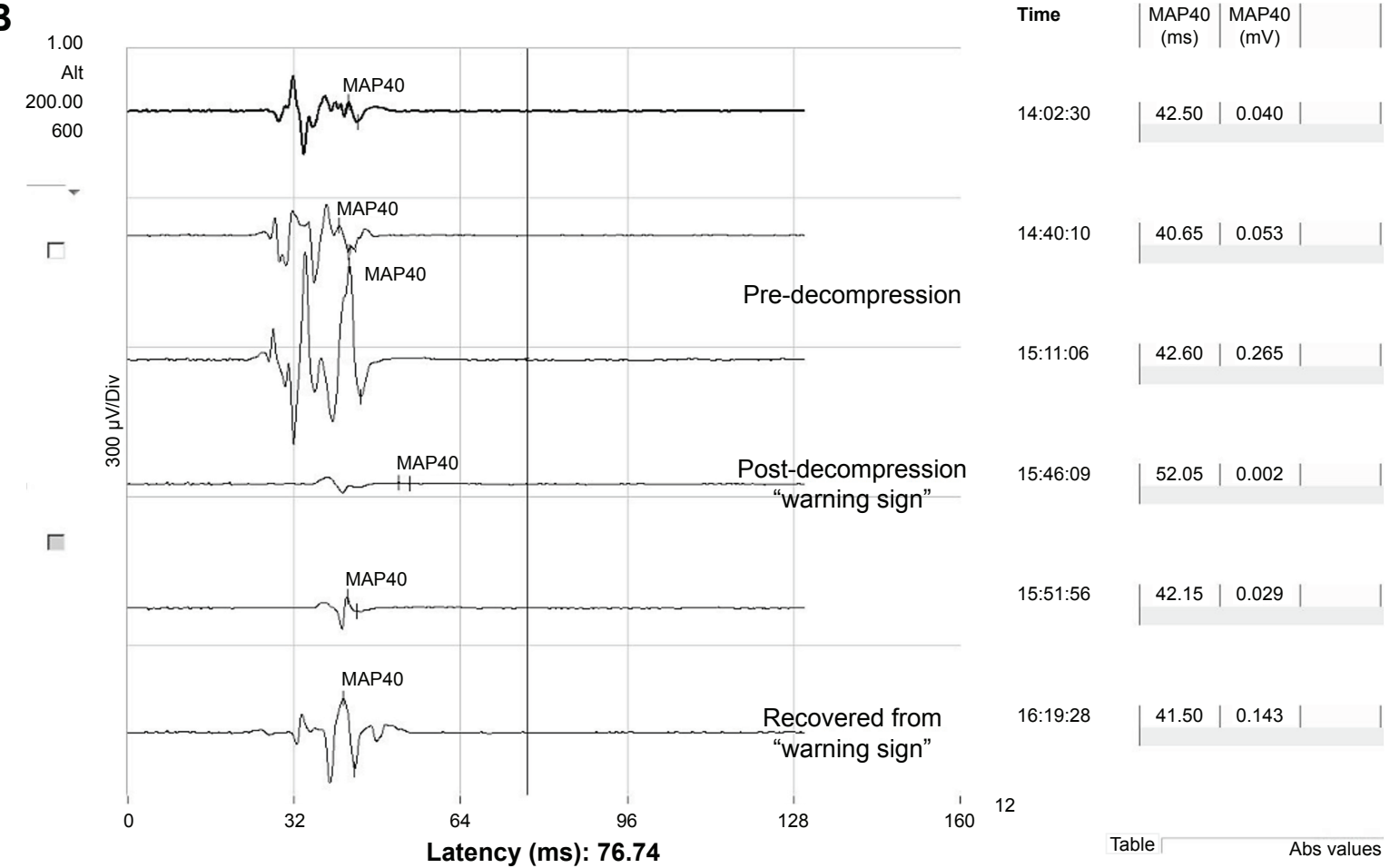

Figure I A case with a "positive change" (A) and a case with "warning sign" after decompression (B) during IOM.

Abbreviations: Abs, absolute; Div, dividend; IOM, intraoperative monitoring.

Similarly, our study reconfirmed the conclusions of previous studies that improvement in MEP during IOM is related to functional improvement 1 month after operation. Although previous studies demonstrated that only MEP amplitude was related to postoperative functional improvement, our study showed that both amplitude and latency of MEP were related to functional improvement. The patients who displayed positive changes in MEP amplitude also displayed positive changes in MEP latency. Monitoring muscle MEP has become a gold standard in surgical assessment because of the increased sensitivity of this technique and the importance of assessment of motor functions during spinal surgery. ${ }^{6}$

Although we evaluated both MEP and SEP during IOM, SEP did not show significant positive changes. This is 
Table I Demographic characteristics of patients

\begin{tabular}{|c|c|c|c|}
\hline Characteristics & $\begin{array}{l}\text { Positive change } \\
\text { group }(n=I I)\end{array}$ & $\begin{array}{l}\text { No positive change } \\
\text { group }(n=18)\end{array}$ & p-value \\
\hline Age & $68.7(10.1)$ & $65.9(7.7)$ & 0.802 \\
\hline $\operatorname{Sex}(M / F)$ & $7 / 4$ & $13 / 5$ & 0.561 \\
\hline Total anesthesia time (min) & $210.7(62.1)$ & $213.8(33.6)$ & 0.631 \\
\hline Total operation time (min) & 147.5 (48.9) & $136.0(28.9)$ & 0.725 \\
\hline Preoperation ASIA motor score $(0-100)$ & $76.3(13.5)$ & $76.9(11.2)$ & 0.613 \\
\hline Preoperation K-MBI (0-100) & $79.2(29.8)$ & $76.4(20.1)$ & 0.852 \\
\hline Hospital stay (day) & $52.5(63.1)$ & I 37.5 (55.7) & 0.030 \\
\hline Neuropathic pain at 6 months after operation, $n(\%)$ & $10(90.9 \%)$ & $16(100 \%)$ & 0.167 \\
\hline
\end{tabular}

Note: Values are the number or mean (SD).

Abbreviations: ASIA, American Spinal Injury Association; K-MBI, Korean version of Modified Barthel Index; M, male; F, female.

consistent with the fact that SEP is slow to respond to spinal decompression, which may be related to the low sensitivity of SEP. ${ }^{18,19}$ Clinical guidelines also recommend that MEP recordings are superior to SEP recordings during spinal cord/spinal column surgery as diagnostic adjuncts for the assessment of spinal cord integrity.

\section{Functional improvement according to the time after operation}

Wang et al evaluated the modified Japanese Orthopedic Association (mJOA) score before and at 6 months after surgery and found that patients with intraoperative MEP improvement after cervical spinal cord decompression experience good neurological recovery both short and long time after surgery. However, our results showed that positive changes in MEP affect functional improvement at 1 month but not at 6 months after surgery. The differences between the previous study and ours may have been due to differences in evaluation tools, namely, the use of the mJOA and K-MBI score, respectively. While the mJOA score is focused on motor power, sensory, and sphincter dysfunction, ${ }^{20-22}$ the K-MBI score evaluates real activities of daily living involving personal hygiene, bathing, feeding, bowel and bladder control, and ambulation. Additionally, K-MBI is widely used to assess functional impairment.
Based on our results, the functional status of patients evaluated using K-MBI was lower at 1 month after operation than that before surgery in both the "positive change" and "no positive change" groups (Figure 2). Various factors, such as preoperative biologic factors, methods of operation, postoperative pain or other complications, and immobilization status, may influence postoperative functional outcomes. Previous studies have revealed that age at the time of presentation, chronicity and severity of myelopathic symptoms, signal changes on preoperative MRI, and their regression/ persistence can be considered predictors of prognosis in patients with CCM. ${ }^{14,23}$

Six months after operation, both groups showed significant improvements in motor power and function relative to those before operation (Figure 2); however, there was no difference in improvement rates between the groups (Figure 3). Thus, in addition to presurgical condition or surgical procedure, proper postoperative management can affect functional improvements after operation. It also suggested that patients without positive changes do not mean definite neurological degeneration or poor outcomes. In addition, regardless of positive changes, nearly all patients suffered from neuropathic pain after cervical spinal cord decompression surgery. Thus, proper postoperative management is important to achieve maximal

Table 2 Changes of IOM pre-decompression and post-decompression

\begin{tabular}{|c|c|c|c|c|c|c|}
\hline \multirow[t]{2}{*}{ Parameter } & \multicolumn{3}{|c|}{ Positive change group $(n=I I)$} & \multicolumn{3}{|c|}{ No positive change group $(n=18)$} \\
\hline & Pre & Post & $p$-value & Pre & Post & $p$-value \\
\hline MEP latency (ms) & 48.35 (7.89) & $4 I .01(5.24)$ & $<0.001$ & $46.09(2.42)$ & $43.34(2.01)$ & 0.182 \\
\hline Amplitude (mV) & $0.14(0.02)$ & $0.29(0.4 \mathrm{I})$ & $<0.001$ & $0.11(0.07)$ & $0.05(0.21)$ & 0.154 \\
\hline SEP latency (ms) & $25.74(5.4 I)$ & $23.49(3.87)$ & 0.065 & $24.87(2.01)$ & $23.18(1.58)$ & 0.723 \\
\hline Amplitude $(\mathrm{mV})$ & $0.787(0.6 \mathrm{I})$ & 0.736 (0.7I) & 0.572 & I.43 (0.79) & I.3I (0.66) & 0.328 \\
\hline
\end{tabular}

Note: Values are the mean (SD).

Abbreviations: MEP, motor evoked potential; SEP, somatosensory evoked potential; IOM, intraoperative monitoring. 
A

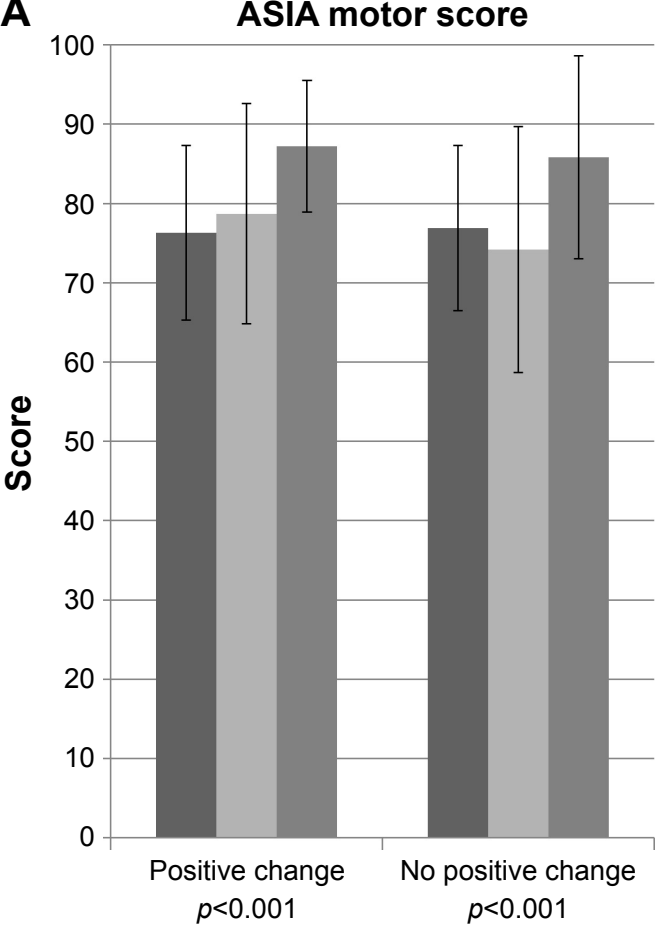

B

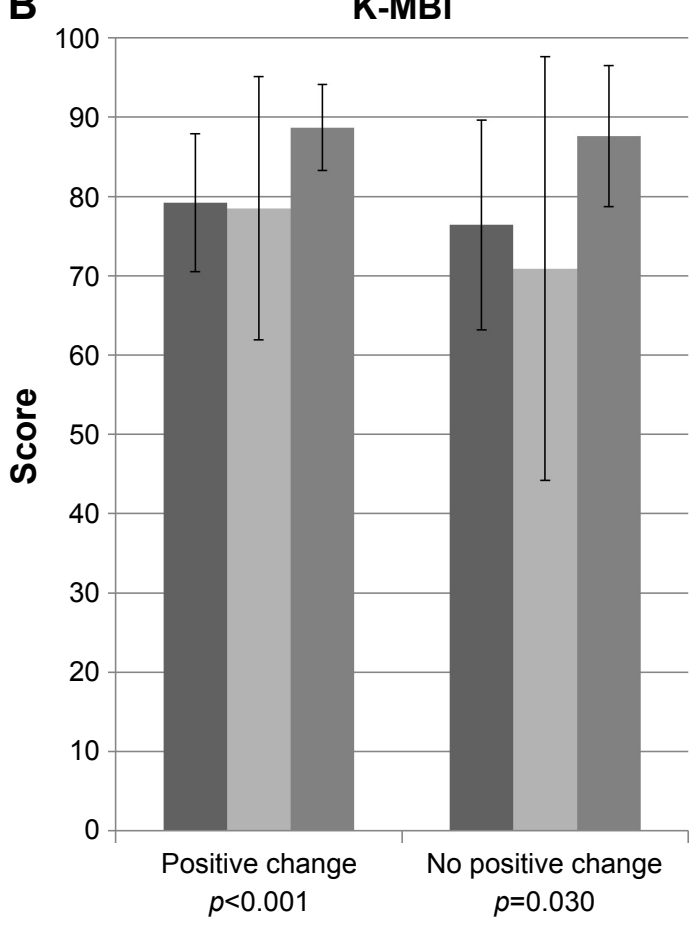

Preop Postop 1 month $\square$ Postop 6 months

Figure 2 Functional changes according to time in both groups.

Notes: In the positive change group, the ASIA motor scale showed significant improvement with time after operation relative to that before operation. However, the no positive change group displayed a slight decrease in motor score at I month after operation relative to that before operation, but the motor score recovered at 6 months after operation (A). K-MBI also decreased at I month after operation relative to that before operation, but it recovered and improved much at 6 months after operation in both groups (B).

Abbreviations: ASIA, American Spinal Injury Association; K-MBI, Korean version of Modified Barthel Index; Preop, preoperative; Postop, postoperative.

functional outcome in patients after cervical spinal cord decompression surgery.

\section{Possible mechanism correlating surgical decompression and changes during IOM}

Several mechanisms can explain the relationship between MEP and functional improvement. One mechanism is that in CCM, nervous tissue of the spinal cord does not undergo necrosis but limits the capability of neurological function; thus, it is reversible through surgical decompression. ${ }^{8}$ In addition, improvements in MEP after neural decompression are probably due to improvements in the excitability of neurons or the corticospinal tract. Anatomically, MEP is used to monitor the corticospinal tract descending from the motor cortex to the anterior horn of the spinal cord and peripheral nerve fibers. ${ }^{24}$ According to previous studies, intraoperative MEP values can be used to detect the functional integrity of the corticospinal tracts with high sensitivity and specificity. ${ }^{9}$ An increase arterial supply can also alleviate spinal cord ischemia and thus result in MEP improvements, ${ }^{8}$ because warning signs can represent a reduction in blood flow during spinal surgery.
SEP represents the function of the dorsal column-lemniscal pathway of the somatosensory system with regard to position, proprioception, and vibration. ${ }^{25}$ Anatomically, dorsal column-lemniscal pathway is located posterior to the corticospinal tract. The dorsal column might be more vulnerable to the compression, and thus, the lack of significant changes in SEP after cervical decompression might be related to the anatomical vulnerability of this region. Previous studies also revealed that MEP changes are more sensitive than SEP changes. ${ }^{5}$ Additionally, because SEP does not represent the spinothalamic tract, which senses pain and changes in temperature, we could not explain the relation between neuropathic pain and changes during IOM.

\section{Limitation}

This study has several limitations including the enrollment of a small number of CCM patients. Additionally, we did not evaluate diffuse tensor images of corticospinal tract or spinothalamic tract. However, we plan to investigate the relationship between diffuse tensor image and functional outcomes in CCM patients in the future. Moreover, we did not use the JOA scale, which is a valid index for evaluating 


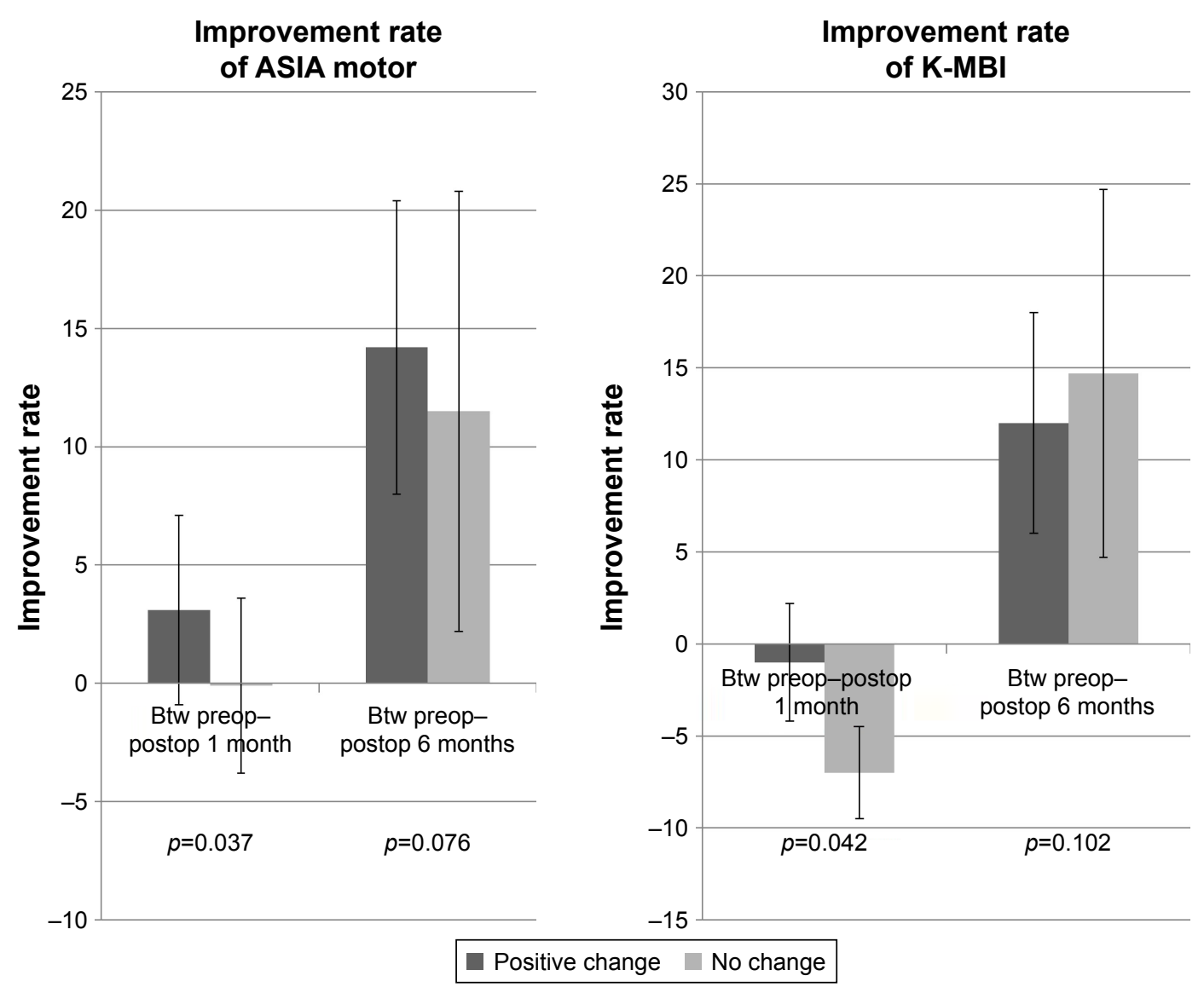

Figure 3 Functional improvement rates in both groups.

Notes: Comparison of improvement rates between the two groups indicated significantly higher ASIA motor score and K-MBI score in patients in the positive change group than in patients in the no positive change group. At one month after surgery, ASIA and K-MBI showed significant improvement rate in the positive change group. But six months after surgery, ASIA and K-MBI showed no significant differences between groups.

Abbreviations: ASIA, American Spinal Injury Association; K-MBI, Korean version of Modified Barthel Index; btw, between; postop, postoperative; preop, preoperative.

neurological outcomes for orthopedic spine surgery. ${ }^{20-22}$ However, K-MBI is sufficient for the evaluation of functional characteristics including gait, activities of daily living, and bowel and bladder functions with the exception of pain. ${ }^{13}$

\section{Conclusion}

Positive changes in MEP during IOM may affect better functional improvement 1 month after operation and early discharge without significant complications in CCM patients. However, positive changes did not affect long-term functional outcomes and neuropathic pain. Thus, proper management is needed to facilitate maximal functional recovery in each patient after cervical spinal decompression surgery.

\section{Author contributions}

SJ Lee: study design, data collection, and manuscript preparation; KW Lee, SB Kim, EY Han, and BR Kim: literature search and data collection; HJ Lee: analysis of data; MK Park: study design and review of manuscript. All Authors contributed toward data analysis, drafting and revising the paper and agree to be accountable for all aspects of the work.

\section{Disclosure}

MK Park was supported by the Dong-A University research fund (No. 2013-2-01). The authors report no other conflicts of interest in this work.

\section{References}

1. Fehlings MG, Tetreault LA, Riew KD, Middleton JW, Wang JC. A clinical practice guideline for the management of degenerative cervical myelopathy: introduction, rationale, and scope. Global Spine J. 2017; 7(3 Suppl):21S-27S.

2. Lubelski D, Alvin MD, Nesterenko S, et al. Correlation of quality of life and functional outcome measures for cervical spondylotic myelopathy. J Neurosurg Spine. 2016;24(3):483-489.

3. Devlin VJ, Schwartz DM. Intraoperative neurophysiologic monitoring during spinal surgery. J Am Acad Orthop Surg. 2007;15(9):549-560.

4. Tamaki T, Kubota S. History of the development of intraoperative spinal cord monitoring. Eur Spine J. 2007;16 Suppl 2:S140-S146.

5. Hadley MN, Shank CD, Rozzelle CJ, Walters BC. Guidelines for the use of electrophysiological monitoring for surgery of the human spinal column and spinal cord. Neurosurgery. 2017;81(5):713-732.

6. Park JH, Hyun SJ. Intraoperative neurophysiological monitoring in spinal surgery. World J Clin Cases. 2015;3(9):765-773.

7. Kim SM, Kim SH, Seo DW, Lee KW. Intraoperative neurophysiologic monitoring: basic principles and recent update. J Korean Med Sci. 2013; 28(9):1261-1269. 
8. Wang S, Tian Y, Wang C, et al. Prognostic value of intraoperative MEP signal improvement during surgical treatment of cervical compressive myelopathy. Eur S J. 2016;25(6):1875-1880.

9. Hilibrand AS, Schwartz DM, Sethuraman V, Vaccaro AR, Albert TJ. Comparison of transcranial electric motor and somatosensory evoked potential monitoring during cervical spine surgery. J Bone Joint Surg Am. 2004;86-A(6):1248-1253.

10. Kirshblum SC, Burns SP, Biering-Sorensen F, et al. International standards for neurological classification of spinal cord injury (revised 2011). J Spinal Cord Med. 2011;34(6):535-546.

11. Marino RJ, Barros T, Biering-Sorensen F, et al. International standards for neurological classification of spinal cord injury. J Spinal Cord Med. 2003;26 Suppl 1:S50-S56.

12. Cuthbert SC, Goodheart GJ Jr. On the reliability and validity of manual muscle testing: a literature review. Chiropr Osteopat. 2007;15:4.

13. Jung HY, Park BK, Shin HS, et al. Development of the Korean Version of Modified Barthel Index (K-MBI): multi-center study for subjects with stroke. J Korean Acad Rehab Med. 2007;31(3):283-297.

14. Pratheesh R, Babu KS, Rajshekhar V. Improvement in intraoperative transcranial electrical motor-evoked potentials in tethered cord surgery: an analysis of 45 cases. Acta Neurochir (Wien). 2014;156(4): $723-731$.

15. Voulgaris S, Karagiorgiadis D, Alexiou GA, et al. Continuous intraoperative electromyographic and transcranial motor evoked potential recordings in spinal stenosis surgery. J Clin Neurosci. 2010;17(2): 274-276.

16. Visser J, Verra WC, Kuijlen JM, Horsting PP, Journee HL. Recovery of TES-MEPs during surgical decompression of the spine: a case series of eight patients. J Clin Neurophysiol. 2014;31(6):568-574.
17. Barley JL, Mooney JF, Glazier SS, et al. Sudden appearance of new upper extremity motor function while performing neurophysiologic intraoperative monitoring during tethered cord release: a case report. $J$ Pediatr Orthop. 2010;30(6):624-628.

18. Schwartz DM, Auerbach JD, Dormans JP, et al. Neurophysiological detection of impending spinal cord injury during scoliosis surgery. J Bone Joint Surg Am. 2007;89(11):2440-2449.

19. Deletis V, Sala F. Intraoperative neurophysiological monitoring of the spinal cord during spinal cord and spine surgery: a review focus on the corticospinal tracts. Clin Neurophysiol. 2008;119(2):248-264.

20. Zhou F, Zhang Y, Sun Y, Zhang F, Pan S, Liu Z. Assessment of the minimum clinically important difference in neurological function and quality of life after surgery in cervical spondylotic myelopathy patients: a prospective cohort study. Eur Spine J. 2015;24(12):2918-2923.

21. Kopjar B, Tetreault L, Kalsi-Ryan S, Fehlings M. Psychometric properties of the modified Japanese Orthopaedic Association scale in patients with cervical spondylotic myelopathy. Spine (Phila Pa 1976). 2015; 40(1):E23-E28.

22. Kalsi-Ryan S, Singh A, Massicotte EM, et al. Ancillary outcome measures for assessment of individuals with cervical spondylotic myelopathy. Spine (Phila Pa 1976). 2013;38(22 Suppl 1):S111-S122.

23. Uchida K, Nakajima $H$, Takeura N, et al. Prognostic value of changes in spinal cord signal intensity on magnetic resonance imaging in patients with cervical compressive myelopathy. Spine J. 2014;14(8):1601-1610.

24. Macdonald DB. Intraoperative motor evoked potential monitoring: overview and update. J Clin Monit Comput. 2006;20(5):347-377.

25. Cruccu G, Aminoff MJ, Curio G, et al. Recommendations for the clinical use of somatosensory-evoked potentials. Clin Neurophysiol. 2008; 119(8):1705-1719.
Clinical Interventions in Aging

\section{Publish your work in this journal}

Clinical Interventions in Aging is an international, peer-reviewed journal focusing on evidence-based reports on the value or lack thereof of treatments intended to prevent or delay the onset of maladaptive correlates of aging in human beings. This journal is indexed on PubMed Central, MedLine,

\section{Dovepress}

CAS, Scopus and the Elsevier Bibliographic databases. The manuscript management system is completely online and includes a very quick and fair peer-review system, which is all easy to use. Visit http://www.dovepress. com/testimonials.php to read real quotes from published authors. 